\title{
Eye manifestations in medullary carcinoma of the thyroid
}

\author{
MOURAD K. KHALIL AND DARIO W. C. LORENZETTI \\ From the Departments of Ophthalmology and Pathology, McGill University, \\ and the Montreal General Hospital, Montreal, Quebec, Canada
}

SUMMARY Multiple endocrine adenomatosis type III (MEA-III) in a 32-year-old white woman is reported. The initial manifestation, a thyroid nodule, was detected at the age of 4 years. The characteristic ocular manifestation of MEA-III are discussed.

We report an interesting case of multiple?endocrine adenomatosis type III (MEA-III), in a 32-year-old white woman in whom the initial manifestation, a thyroid nodule, was detected at the age of 4 years. The characteristic ocular manifestations of MEAIII will be discussed as well as the role of the ophthalmologist in the early diagnosis of this syndrome.

\section{Case history}

The patient was a 32-year-old white woman. In 1951, when she was 4 years old, her mother noted a lump in her neck which was subsequently diagnosed as a nodule in the left lobe of her thyroid gland. Repeated physical examinations over the ensuing years revealed many signs involving different systems, eventually suggesting the presence of a complex syndrome. These included prognathism, thick lips, poor dentition, a large tongue with multiple marginal nodules, multiple polyps of the vocal cords, and a marfanoid habitus with long extremities, arachnodactyly, hyperextensibility, and increased laxity of the joints. In 1961 and 1962 she had operations for slipped right and left femoral epiphyses. She also suffered from recurrent bouts of severe diarrhoea.

Laboratory investigations during these years showed no thyroid dysfunction, a normal sella turcica, and normal levels of growth hormone.

In 1966 an excisional biopsy of the thyroid nodule was performed and histopathologically was diagnosed as Hurtle-cell carcinoma. This was followed by a total thyroidectomy. A later review of these pathological specimens established the correct

Correspondence to Dr M. Khalil, Department of Ophthalmology, Room 4813, Livingston Hall, Montreal General Hospital, 1650 Cedar Avenue, Montreal, P.Q., Canada H3G 1 A4.

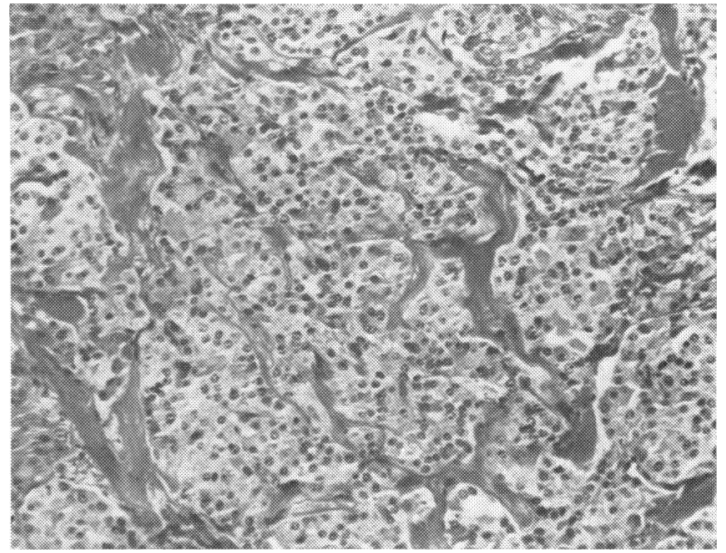

Fig. 1 Histopathology of medullary carcinoma of the thyroid. Parafollicular cells and stromal amyloid $(\times 18)$.

diagnosis of medullary carcinoma of the thyroid gland (Fig. 1). Once this diagnosis was established, investigations were carried out to check for other manifestations of multiple endocrine neoplasia. Our patient showed an absence of flare reflex after intradermal histamine test, normal serum calcium, and an absence of pheochromocytomas. There was no relevant family history. In 1967 mandibular osteotomies and dental repair operations were carried out, though residual prognathism remained (Fig. 2). A tongue nodule was also biopsied and it displayed the typical histopathology of a neuroma (Fig. 3).

In 1971 an enlarged cervical lymph node, noted on a routine examination, was excised, and it was negative for metastatic disease. A chest $x$-ray, skeletal survey, and serum calcium levels were again normal, suggesting that there were no metastases.

We first examined the patient in 1973. The visual 
Fig. 2 Thick lips and residual prognathism following mandibilar osteotomies.
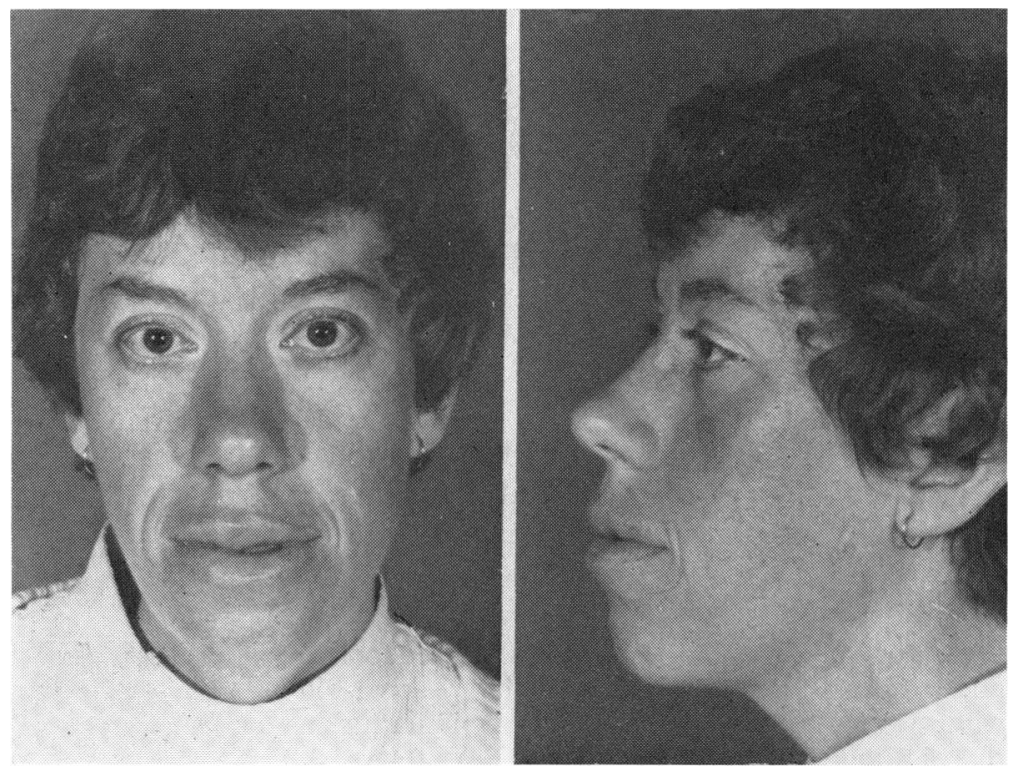

acuity was normal in both eyes. She had thick everted lid margins with anterior displacement of the cilia and ectropion puncti. The conjunctival edge of the lid margins was studded with small

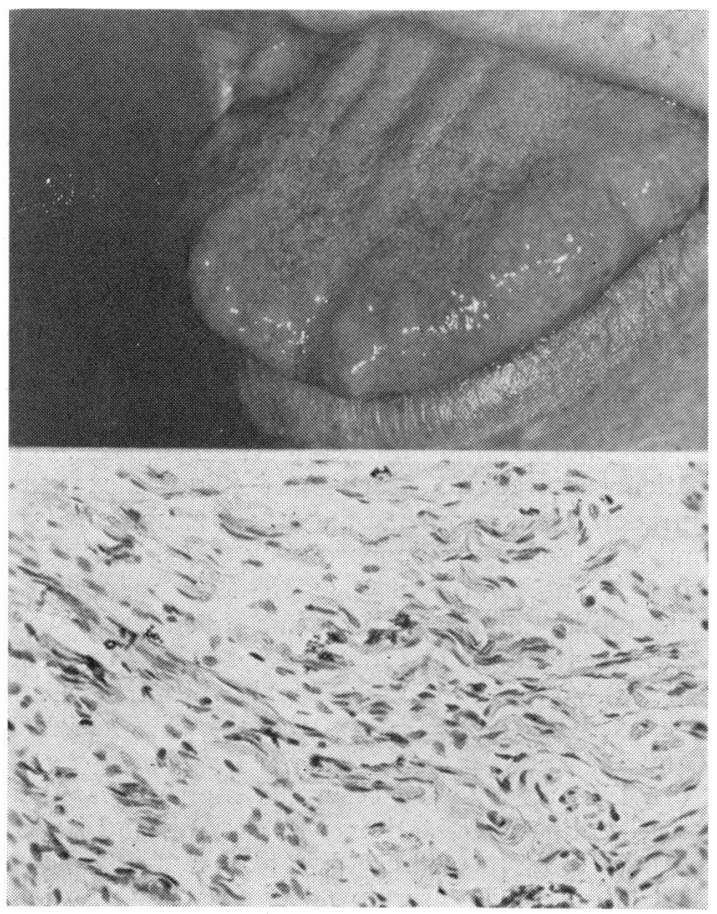

Fig. 3 Marginal nodules of the tongue. Histopathology of a tongue nodule showing a typical neuroma $(\times 34)$. irregular nodules (Fig. 4), and the bulbar conjunctiva contained diffuse swellings superior to the limbus bilaterally. There were bilateral thickened opaque corneal nerve fibres (Fig. 5). The patient also had dry eyes bilaterally as determined by repeated Schirmers testing. Excisional biopsies of both the conjunctival nodules on the lid margins and the superior bulbar swellings displayed the typical histopathology of plexiform neuromas (Fig. 6).

For several years she complained of occasional episodes of palpitations and sweating, though she never had any recorded hypertension. In 1976 pheochromocytomas were diagnosed by raised 24-hour urinary vanillyl mandelic acid (VMA) test

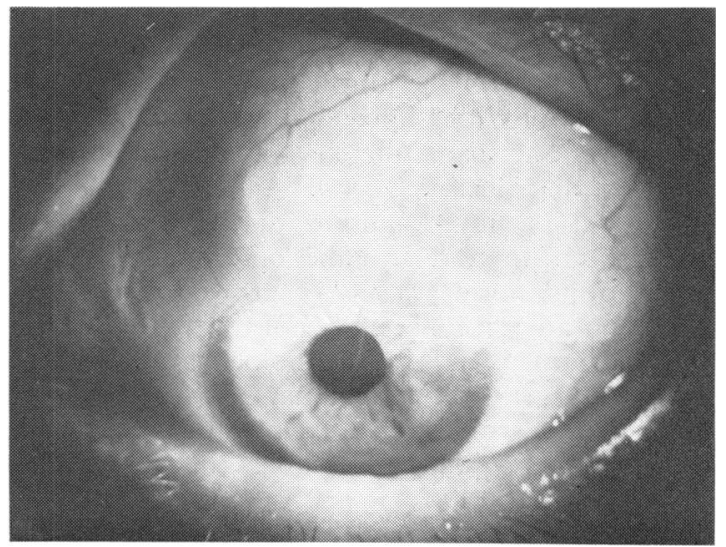

Fig. 4 Thickened lid margin with multiple nodular irregularities on the conjunctival edge. 


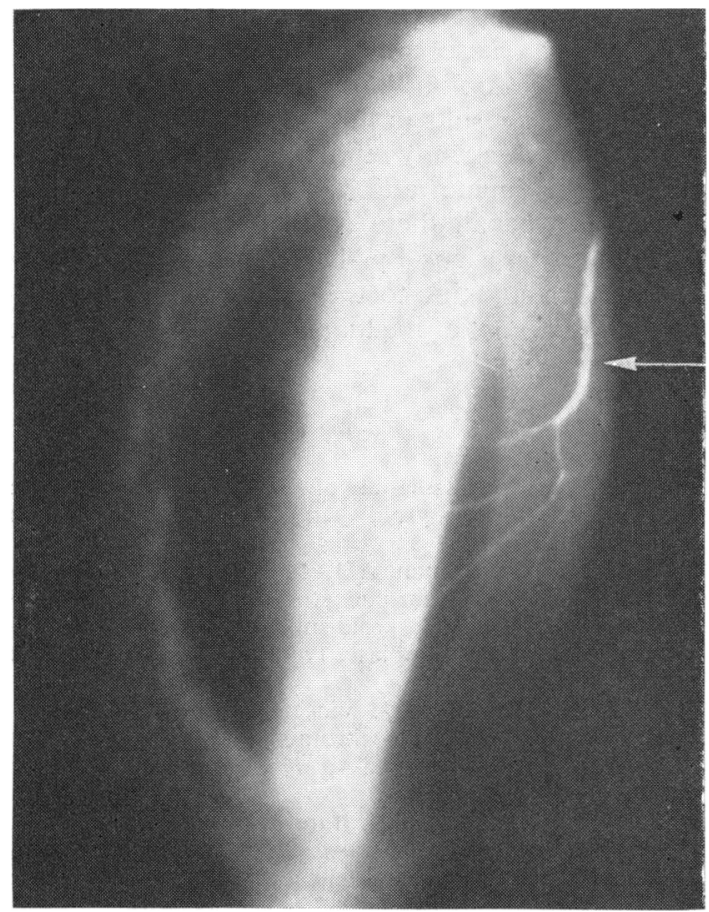

Fig. 5 Slit-lamp photograph showing thickened opaque corneal nerves.

and by a positive glucagon test. Adrenal venography confirmed the presence of a moderate sized tumour of the left adrenal and a possible abnormality of the right. In 1977 she underwent bilateral adrenalectomy and a cholecystectomy. The right adrenal contained a small pheochromocytoma while the left was markedly enlarged with this tumour. The gall bladder contained numerous calculi, and histopathologically there was a gangliomatosis of the gall bladder wall.

\section{Discussion}

The term multiple endocrine neoplasia (MEN) has been used since 1932, although Sipple ${ }^{1}$ in 1961 first established the association between familial medullary carcinoma of the thyroid and pheochromocytomas. Later Steiner ${ }^{2}$ divided MEN into types I and II, and Gorlin ${ }^{3}$ subdivided type II into A and B. Then in 1975 Khairi $^{4}$ suggested that type IIB be called type III. Finally the term multiple endocrine adenomatosis (MEA) was suggested as a better term for this group of syndromes.

Pertinent features of these 3 syndromes are as follows. ${ }^{5}$

$M E A$-type I (Wermer's syndrome). Acidophilic and chromophobic adenomas of the pituitary gland, functioning islet-cell tumours of the pancreas, adenomas of the parathyroid and adrenal cortex: and intractable peptic ulcer. Ocular symptoms are secondary to the pituitary tumours.

MEA-type II (Sipple's syndrome). Medullary carcinoma of the thyroid, pheochromocytomas, and parathyroid hyperplasia and adenomas.

MEA-I and II are two genetically distinct tumour endocrinopathies which both show autosomal dominant inheritance, and little overlap exists between them. MEA-II appears to be a form of neuroectodermal dysplasia, and the pheochromocytomas are of neural crest origin. A substantial body of evidence supports a similar derivation for the parafollicular cells (C cells) of the thyroid. A variant of MEA-II is the association of mucosal neuromas, which are also derivatives of the neural crest. This variant is designated by some authors as MEA-IIB and by others as MEA-III. Most cases with associated multiple mucosal neuromas are reported to be sporadic.

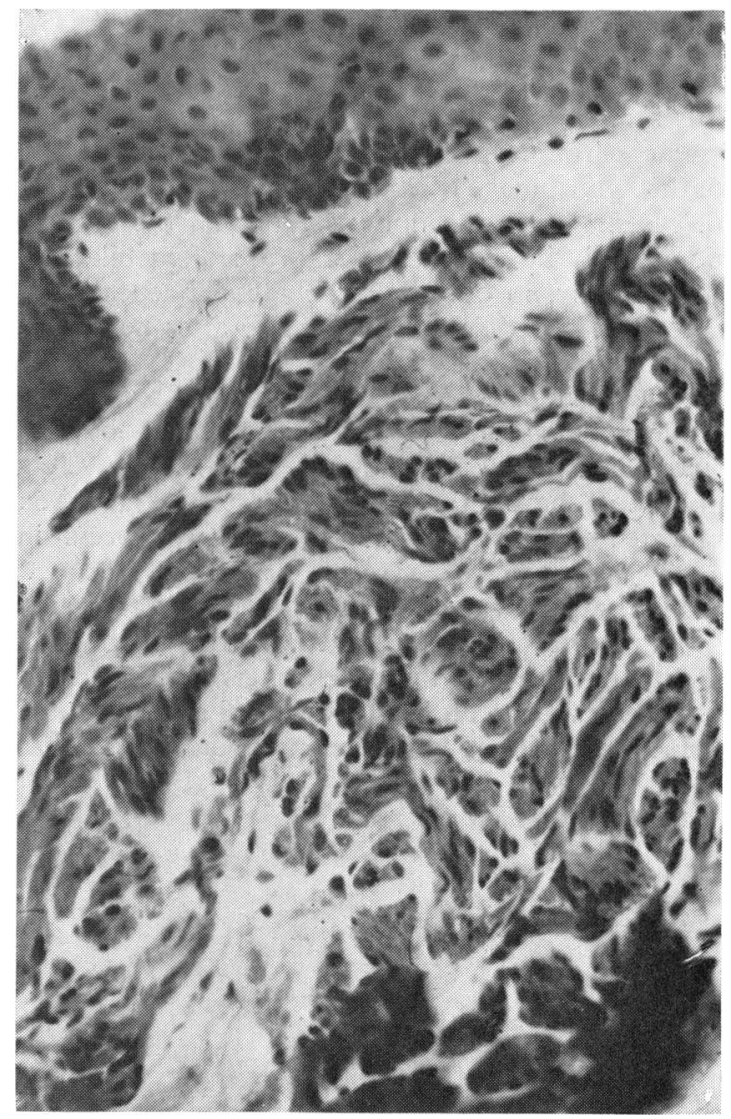

Fig. 6 Histopathology of a plexiform neuroma of the bulbar conjunctiva $(\times 40)$. 
$M E A-I I I$. Medullary carcinoma of the thyroid, parathyroid usually normal or hyperplastic, pheochromocytomas, multiple mucosal neuromas and skeletal manifestations suggestive of Marfan's syndrome. Ophthalmic findings include multiple neuromas of the lids and conjunctivae, dry eyes, and opaque nerves of the cornea. Patients have characteristic facies due to thick patulous lips, soft tissue prognathism, and large tongues studded with multiple nodules (neuromas).

Our case represents a MEA-III syndrome in which there was no family history. It is interesting that the thyroid nodule was first detected at age 4, at which time the clinical pathological entity of medullary carcinoma of the thyroid was not yet established. Only in 1959 did Hazard et al. ${ }^{6}$ differentiate medullary carcinoma from other types of thyroid carcinoma by the presence of stromal amyloid. The origin of medullary carcinoma has been established to be the parafollicular cells (C cells), which are the calcitonin-secreting cells. Calcitonin studies are therefore diagnostic and are mandatory in any individual with suspected medullary carcinoma of the thyroid. Detailed descriptions of the systemic, ocular, and laboratory features of this syndrome have been previously reported. ${ }^{7-9}$

Pheochromocytomas are not a constant feature of this syndrome (MEA-III) but are present in $37 \%$ of cases. ${ }^{10}$ They are more a feature of the familial dominantly inherited cases and are often bilateral. Interestingly, in our patient bilateral pheochromocytomas were diagnosed 28 years after her initial investigation of this syndrome.

Medullary carcinomas of the thyroid are of low grade malignancy. Our patient has been observed since 1951, when her thyroid nodule was discovered, and there have been no metastases. It has been reported that lymph node metastases do occur in $48-70 \%$ of cases.

Mucocutaneous neuromas are an integral feature of 'multiple endocrine adenomatosis' type III. Ophthalmologically these neuromas occur as convoluted swellings or nodules of the bulbar and palpebral conjunctiva. The many nodular neuromas of the palpebral conjunctiva may thicken the lid margins and cause anterior displacement of the lashes and ectropion puncti. Involvement of the corneal nerves results in thick opaque nerve fibres. ${ }^{11}$ Dry eyes are frequently reported and are supposedly due to inadequate autonomic innervation. Our patient showed all these characteristics. Detection of these ocular signs by the ophthalmologist may provide the only clue to the diagnosis of an otherwise asymptomatic medullary carcinoma of the thyroid.

\section{References}

1 Sipple JH. The association of pheochromocytoma with carcinoma of the thyroid gland. Am J Med 1961; 31: 163-6.

2 Steiner AL, Goodman AD, Powers SR. Study of a kindred with pheochromocytoma, medullary thyroid carcinoma, hyperparathyroidism and Cushing's diseasemultiple endocrine neoplasia type 2. Medicine 1968; 47: 371-409.

3 Gorlin RJ, Sedano HO, Vickers RA, et al. Multiple mucosal neuromas, pheochromocytoma and medullary carcinoma of the thyroid-a syndrome. Cancer 1968; 22: 293-9.

4 Khairi MRA, Dexter RN, Burzynski NJ, Johnston CC. Mucosal neuroma, pheochromocytoma and medullary thyroid carcinoma: multiple endocrine neoplasia-type III. Medicine 1975; 54: 89-112.

5 Schimke RN. Multiple endocrine adenomatosis syndromes. Adv Intern Med 1976; 21 : 249-65.

6 Hazard JB, Hawk WA, Crile G. Medullary (solid) carcinoma of the thyroid-a clinicopathologic entity. J Clin Endocrinol 1959; 19: 152-61.

7 Baum JL, Adler ME. Pheochromocytoma, medullary thyroid carcinoma, multiple mucosal neuroma. Arch Ophthalmol 1972; 87: 574-84.

8 Colombo CG, Watson AG. Ophthalmic manifestations of multiple endocrine neoplasia, type three. Can Ophthalmol 1976; 11: $290-4$.

9 Hazard JB. The C-cells (parafollicular cells) of the thyroid gland and medullary thyroid carcinoma-a review. Am J Pathol 1977; 88: 214-49.

10 Schimke RN, Hartmann WH, Prout TE, et al. Syndrome of bilateral pheochromocytoma, medullary thyroid carcinoma and multiple neuromas. $N$ Engl $J$ Med 1968; 279: 1-7.

11 Robertson DM, Sizemore GW, Gordon H. Thickened corneal nerves as a manifestation of multiple endocrine neoplasia. Trans Am Acad Ophthalmol Otolarngol 1975; 79: 772-87. 